\title{
A predictor for mitral valve repair in patient with rheumatic heart disease: the bending angle of anterior mitral leaflet
}

\author{
Jin-Tao Fu, Mohammad Sharif Popal, Yu-Qing Jiao, Hai-Bo Zhang, Shuai Zheng, Qiu-Ming Hu, Wei \\ Han, Xu Meng
}

Department of Cardiac Surgery, Beijing Anzhen Hospital, Capital Medical University, Beijing 100029, China

Contributions: (I) Conception and design: JT Fu, X Meng; (II) Administrative support: X Meng; (III) Provision of study materials or patients: JT Fu, MS Popal, YQ Jiao; (IV) Collection and assembly of data: JT Fu, MS Popal, YQ Jiao, W Han; (V) Data analysis and interpretation: JT Fu ,S Zheng, QM Hu; (VI) Manuscript writing: All authors; (VII) Final approval of manuscript: All authors.

Correspondence to: Xu Meng. Department of Cardiac Surgery, Beijing Anzhen Hospital, No. 2 Anzhen Road ,Chaoyang District, Beijing 100029, China. Email: mxu@263.net.

Background: Mobility and flexibility of anterior mitral leaflet (AML) are considered to be the important factor for mitral valve (MV) repair in rheumatic population, we try to use the bending angle of AML to quantify its' mobility and flexibility and investigate whether it can predict the success of rheumatic MV repair or not.

Methods: Total 54 patients underwent rheumatic MV surgeries with mild lesions of subvalvular apparatus from August, 2017 to November, 2017 at the author institution, we divided the patients into MV repair and MV replacement groups which included directly MV replacement and repair attempt but failed repairing transfer to replacement intraoperatively. Patients' MV structure was carefully evaluated on transthoracic echocardiography (TTE) and scored by the Wilkins score (WS) preoperatively. The bending angle of AML was measured during systole and diastole at different level before surgery.

Results: The differences of patients' demographic characteristics between repair group and replacement group were not statistically significant $(\mathrm{P}$ value $>0.05)$ and the differences among B-angle, T-angle, Bs-angle, $\mathrm{C}$-angle of AML between repair group and replacement groups were not statistically significant $(\mathrm{P}$ value $>0.05)$. Only BT-angle in repair group was significantly larger than that of replacement group $\left(21.56^{\circ} \pm 3.84^{\circ}\right.$, $10.29^{\circ} \pm 6.02^{\circ}$, respectively, $\mathrm{P}<0.001$ ), therefore the BT-angle was tested as a predictor of reparability by observing the receiver operating characteristic (ROC) curve (ROC area: 0.944, standard error: 0.06, 95\% CI: $0.826-1$ ), BT-angle of AML on preoperative transthoracic echo of $15.5^{\circ}$ or more predicts feasibility of rheumatic MV repair with $100 \%$ sensitivity and $85.7 \%$ specificity in patients with mild lesions of subvalvular apparatus. Logistic regression for a single area of calcification (diameter $<0.8 \mathrm{~cm}$ ) at commissure were shown that: $\beta: 0.08, \operatorname{Exp}(\beta): 1.083$, P: $0.777>0.05$, the minor single calcified area at commissure had no effect on reparability of rheumatic MV disease. The differences of total WS and each component score between repair group and replacement group were not statistically significant ( $\mathrm{P}$ value $>0.05$ ). By observing the ROC curve for total WS (ROC area: 0.508), the WS cannot be a predictor for the rheumatic MV repair.

Conclusions: The bending angle of AML which was considered as quantification of mobility and flexibility of AML can be a predictor for MV repair in patients with rheumatic heart disease (RHD). The small single area of commissural calcification had no effect on reparability of rheumatic MV disease. WS cannot appropriately predict the outcome of rheumatic MV repair.

Keywords: Predictor; mitral valve (MV) repair; rheumatic heart disease (RHD); angle of anterior mitral leaflet (AML)

Submitted Feb 07, 2018. Accepted for publication Apr 17, 2018.

doi: $10.21037 /$ jtd.2018.05.26

View this article at: http://dx.doi.org/10.21037/jtd.2018.05.26 


\section{Introduction}

Rheumatic mitral valve (MV) disease is the largest constituent of valvular heart disease in developing countries, meanwhile it is one of the major health concerns universally $(1,2)$. It is resulted from the acute rheumatic fever and the entire MV complex can be affected adversely (3). MV repair is an optimal surgical strategy for such patients when it is compared with MV replacement $(4,5)$, however, MV repairing surgery is not always successful due to the severity of affected MV. Chronic inflammation and fibrosis can result in thickening and restrictive mobility of mitral leaflet. Mobility, length of anterior mitral leaflet (AML) (6), subvalvular apparatus lesion, and calcification may play a crucial role for the decision of rheumatic MV repair or replacement. In our previous experience, the bending angel of AML during systolic and diastolic period can be the symbol of flexibility and mobility of AML. Transthoracic echocardiography (TTE) should be obtained firstly when valvular heart disease is suspected (7) and measurement of the bending angle of AML can be acquired preoperatively. Although Wilkins score (WS) (8) is classic and predominantly utilized scoring system to select appropriate patients for the percutaneous mitral balloon valvuloplasty (PMBV) in patients with mitral stenosis (MS), it is also an effective evaluative method for leaflet mobility, subvalvular thickening, leaflet thickening and calcification. In the current study, we tried to utilize bending angle of AML to quantify the mobility and flexibility of AML, also investigated if the bending angle of AML is a predictive factor for immediate successful MV repair in patients with rheumatic heart disease (RHD), and can WS predict the feasibility of rheumatic MV repair? Is it appropriate evaluation method for percutaneous balloon dilatation in MS or not?

\section{Methods}

\section{Patients' demographic characteristics and clinical features}

Total 78 consecutive patients with RHD underwent MV surgeries from August, 2017 to November, 2017 based on 2014 AHA/ACC and 2017 AHA/ACC Guidelines $(9,10)$ for the Management of Patients With Valvular Heart Disease, individual features and clinical indicators. The MV structure of all the patients were carefully evaluated by TTE preoperatively, patients that underwent simultaneous cardiac surgery for coronary artery disease and had history of myocardial infarction were excluded. Eventually, 54 patients which had mild lesions of subvalvular apparatus were enrolled in this study. We defined directly MV replacement and repair attempt but failed repairing transfer to replacement intraoperatively and counted as a replacement surgery, the enrolled patients were placed into repair and replacement groups. The patients' demographic characteristics are listed in Table 1.

\section{Echocardiographic evaluation}

Patients' MV structure was carefully evaluated on TTE and scored by the WS preoperatively. The bending angles of AML was measured during systole and diastole at different level before the surgery. All data was measured and analyzed twice by two independent echocardiography specialists (YQ Jiao and W Han) blinded to each other's results. The angles between a line connecting the two leaflet origin at the annulus to the bending- and tippoint of AML in end-diastole were defined as B-angle and $\mathrm{T}$-angle, both of them are maximum motion in diastole, the measurement of AML angle in diastole is illustrated in Figure 1. The angles between a line connecting the two leaflet origin at the annulus to the bending- and coaptation-point of AML in end-systole were defined as Bs-angle and C-angle, The measurement of AML angle in systole is illustrated in Figure 2. The angle between a line connecting the leaflet origin at the annulus to the bendingpoint and a line connecting the leaflet origin at the annulus to tip-point of AML in end-diastole is defined BT-angle (Figure 3)

Angles of AML were measured on TTE preoperatively and evaluated by each scoring component of WS, the angles and WS were compared in both group which were listed in Table 2.

\section{Surgical strategies}

All the patients underwent rheumatic MV repair or replacement with median sternotomy access. All procedures were carried out by the same experienced chief surgeon ( $\mathrm{X}$ Meng). Several repair techniques were performed related to the pathological features and severity of the cases, these techniques included: leaflet thinning, commissurotomy, decalcification, papillary muscle splitting, chordal transfer, ring annuloplasty. We used water test and transesophageal echocardiography to examine the immediately effect of MV repair, patients who had more than a moderate residual mitral regurgitation (MR) after repair were intervened 
with MV replacement intraoperatively, and the patients who were not suitable candidates for MV repair after the assessment underwent MV replacement respectively. The detailed repair techniques and the intraoperative result after rheumatic MV repair on transesophagus echocardiography

Table 1 Patients' demographic characteristics

\begin{tabular}{|c|c|c|c|}
\hline Indexs & $\begin{array}{l}\text { Repair group } \\
\qquad(\mathrm{n}=28)\end{array}$ & $\begin{array}{l}\text { Replacement } \\
\text { group }(n=26)\end{array}$ & $P$ value \\
\hline Gender-female & 24 & 20 & 0.406 \\
\hline Age & $53.11 \pm 9.83$ & $58.14 \pm 8.57$ & 0.302 \\
\hline Hypertension & 6 & 8 & 0.434 \\
\hline Diabetes & 5 & 6 & 0.634 \\
\hline AF & 20 & 19 & 0.893 \\
\hline TR & 22 & 23 & 0.330 \\
\hline AV disease & 4 & 5 & 0.626 \\
\hline \multicolumn{4}{|c|}{ Pathological feature } \\
\hline Mixed lesion & 14 & 14 & 0.777 \\
\hline Pure MS & 14 & 12 & 0.777 \\
\hline SAIEBC & 2 & 2 & 0.939 \\
\hline $\operatorname{MVOA}\left(\mathrm{cm}^{2}\right)$ & $1.14 \pm 0.47$ & $1.31 \pm 0.41$ & 0.474 \\
\hline $\operatorname{MRA}\left(\mathrm{cm}^{2}\right)$ & $4.43 \pm 4.39$ & $8.24 \pm 7.13$ & 0.208 \\
\hline LVEF (\%) & $58.89 \pm 3.14$ & $59.29 \pm 5.345$ & 0.855 \\
\hline
\end{tabular}

$P$ value $<0.05$ means the difference is statistical significant. AF, atrial fibrillation; TR, tricuspid regurgitation; $A V$, aortic valve; MS, mitral stenosis; SAIEBC, single area of increased echo brightness (diameter $<0.8 \mathrm{~cm}$ ) at commissure; MVOA, mitral valve orifice area; MRA, mitral regurgitation area; LVEF, left ventricular ejection fraction.
(TEE) are listed in Table 3.

\section{Statistical analysis}

Continuous or interval-related variables were expressed as mean \pm standard deviation (SD). For comparison of variables between the two groups Student's $t$-test was utilized. Univariate analysis of variables was performed with the $\chi^{2}$ or Fisher's exact tests. Receiver operating characteristic (ROC) curves was utilized to define the best cutoff value that was associated with outcomes. Logistic regression was used to determine the predictors associated with the outcomes of rheumatic MV repair. $\mathrm{P}$ value $<0.05$ was considered statistically significant. All statistical analysis was performed via the SPSS 21.

\section{Results}

Patients' demographic characteristics in repair group have been compared with replacement group, the differences of clinical and echocardiography features between repair group and replacement group were not statistically significant (all the $\mathrm{P}$ value $>0.05$ ), there were 4 patients with small single area of increased echo brightness (diameter $<0.8 \mathrm{~cm}$ ) at commissure on preoperative TTE in the two groups (each group has 2 patients, diameter range from 0.4 to $0.8 \mathrm{~cm}$ ), those are revealed in Table 1 . Postoperative MRA was significantly less than before the repair surgery $\left(0.6 \pm 0.16 \mathrm{~cm}^{2}, 4.43 \pm 4.39 \mathrm{~cm}^{2}\right.$, respectively, $\left.\mathrm{P}<0.001\right), \mathrm{MV}$ orifice area after repair surgery was significantly larger than preoperative orifice area $\left(2.27 \pm 0.12 \mathrm{~cm}^{2}, 1.14 \pm 0.47 \mathrm{~cm}^{2}\right.$, respectively, $\mathrm{P}<0.05)$.

Different angles of AML in both groups have been
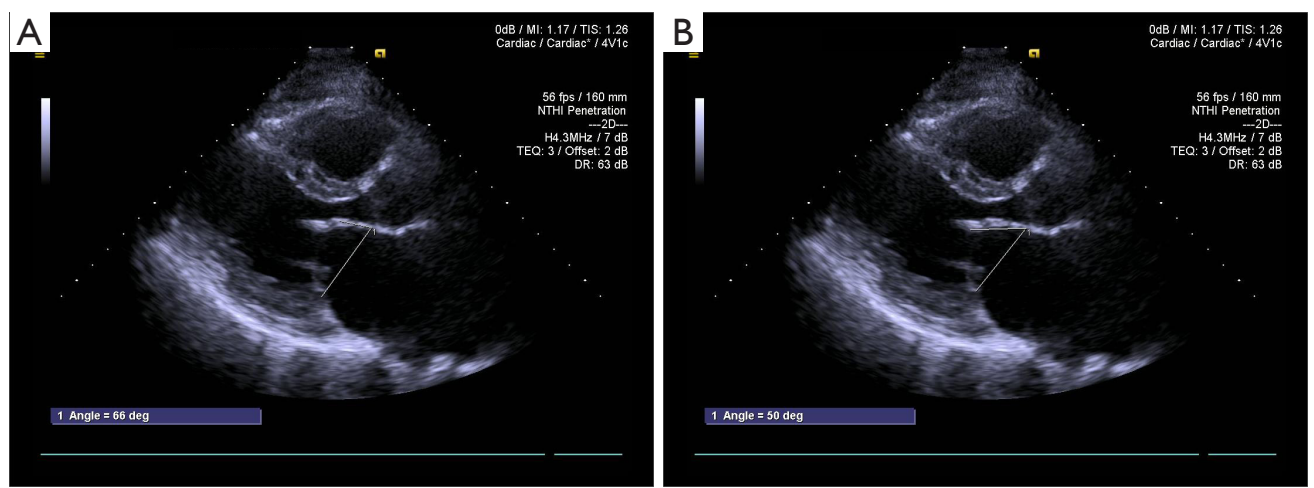

Figure 1 The angles between a line connecting the two leaflet origin at the annulus to the bending- and tip-point of AML in end-diastole were defined as B-angle (A) and T-angle (B). AML, anterior mitral leaflet. 

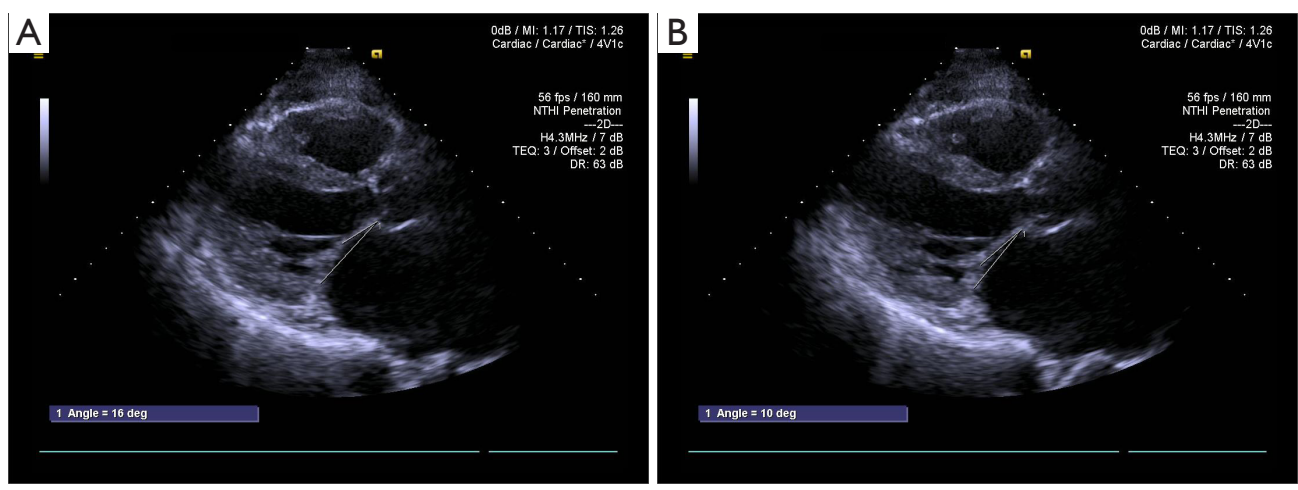

Figure 2 The angles between a line connecting the two leaflet origin at the annulus to the bending- and coaptation-point of AML in endsystole were defined as Bs-angle (A) and C-angle (B). AML, anterior mitral leaflet.
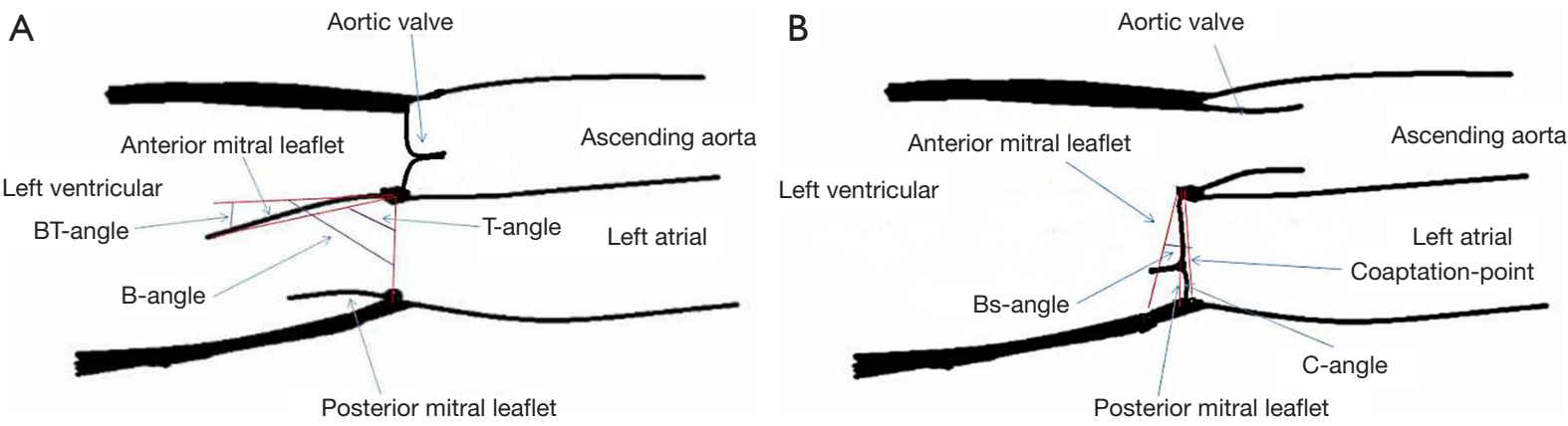

Figure 3 BT-angle: the angle between a line connecting the leaflet origin at the annulus to the bending-point and a line connecting the leaflet origin at the annulus to tip-point of AML in end-diastole (A). (A) and (B) illustrating the 5 different angles of AML. AML, anterior mitral leaflet.

Table 2 Patients' angles on TTE and WS

\begin{tabular}{lccc}
\hline Indexs & $\begin{array}{c}\text { Repair } \\
\text { group }\end{array}$ & $\begin{array}{c}\text { Replacement } \\
\text { group }\end{array}$ & P value \\
\hline B-angle $\left(^{\circ}\right)$ & $64 \pm 5.48$ & $58.14 \pm 9.09$ & 0.131 \\
T-angle $\left(^{\circ}\right)$ & $42.22 \pm 4.94$ & $47.57 \pm 6.99$ & 0.094 \\
BT-angle $\left(^{\circ}\right)$ & $21.56 \pm 3.84$ & $10.29 \pm 6.02$ & 0.001 \\
Bs-angle $\left(^{\circ}\right)$ & $17 \pm 15.63$ & $20.57 \pm 16.69$ & 0.666 \\
C-angle $\left(^{\circ}\right)$ & $14.11 \pm 9.51$ & $13.86 \pm 8.19$ & 0.956 \\
WS (total) & $8.11 \pm 0.78$ & $8.14 \pm 1.22$ & 0.950 \\
Mobility & $2.22 \pm 0.44$ & $2.29 \pm 0.49$ & 0.789 \\
Subvalvular thickening & $2.67 \pm 0.5$ & $2.14 \pm 0.69$ & 0.099 \\
Thickening & $2.11 \pm 0.6$ & $2.29 \pm 0.49$ & 0.543 \\
Calcification & $1.11 \pm 0.6$ & $1.29 \pm 0.95$ & 0.660 \\
\hline
\end{tabular}

$P$ value $<0.05$ means the difference is statistical significant. TTE, transthoracic echocardiography; WS, Wilkins score.
Table 3 Repair techniques and comparison of MRA and MVOA before and after rheumatic MV repair

\begin{tabular}{lcc}
\hline Items & $\mathrm{N}$ & P value \\
\hline Repair technique & 28 & \\
Commissurotomy & 7 & \\
Papillary muscle splitting & 2 & \\
decalcification & 21 & \\
Leaflet thinning & 28 & \\
Ring annuloplasty & 4 & \\
Chordal transfer & & \\
MRA (cm ${ }^{2}$ ) & $4.43 \pm 4.39$ & \\
Pre-operation & $0.6 \pm 0.16$ & \\
Post-operation & & \\
MVOA (cm ${ }^{2}$ ) & $1.14 \pm 0.47$ & \\
Pre-operation & $2.27 \pm 0.12$ & \\
Post-operation &
\end{tabular}

$P$ value $<0.05$ means the difference is statistical significant. MRA: mitral regurgitation area; MVOA: mitral valve orifice area; $\mathrm{MV}$, mitral valve. 


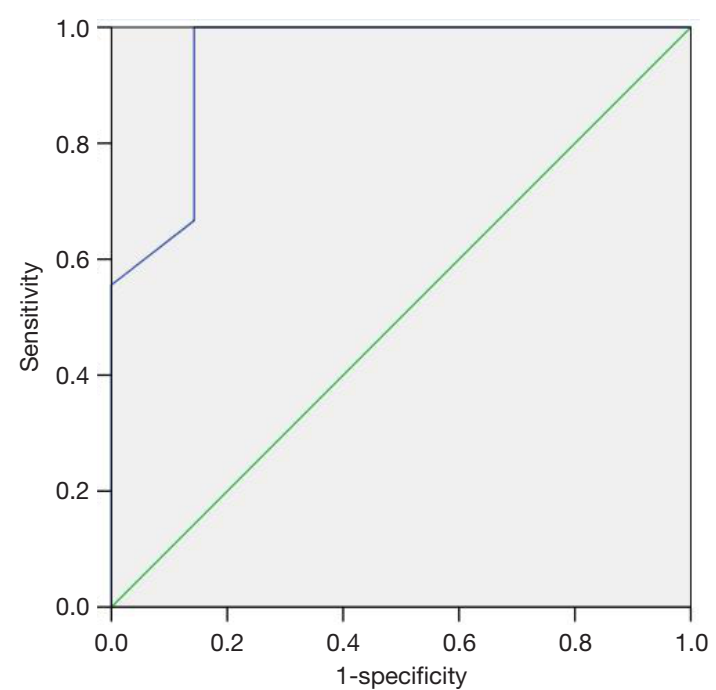

Figure 4 Receiver operating characteristic (ROC) curve for BTangle of anterior mitral leaflet (AML).

measured before the surgery. The differences of B-angle, T-angle, Bs-angle, $\mathrm{C}$-angle between repair group and replacement group were not statistically significant $(\mathrm{P}$ value $>0.05)$. Only BT-angle in repair group was significantly larger than that of replacement group $\left(21.56^{\circ} \pm 3.84^{\circ}\right.$, $10.29^{\circ} \pm 6.02^{\circ}$, respectively, $\mathrm{P}<0.001$ ) (Table 2 ), therefore the BT-angle was tracked as a predictor of reparability by observing the ROC curve (Figure 4) (ROC area: 0.944, standard error: $0.06,95 \% \mathrm{CI}$ : 0.826-1), BT-angle on TTE preoperatively of $15.5^{\circ}$ or more predicts feasibility of rheumatic MV repair with $100 \%$ sensitivity and $85.7 \%$ specificity in patients with mild lesions of subvalvular apparatus. Logistic regression for a single area of increased echo brightness (diameter $<0.8 \mathrm{~cm}$ ) at commissure was expressed as: $\beta$ : $0.08, \operatorname{Exp}(\beta): 1.083, P: 0.777>0.05$, the single area of increased echo brightness (diameter $<0.8 \mathrm{~cm}$ ) at commissure had no effect on reparability of rheumatic MV disease.

The differences of total WS score and each component score between repair group and replacement group were not statistically significant $(\mathrm{P}>0.05)$ (Table 2). By viewing the ROC curve for total WS score (ROC area: 0.508), the WS cannot be a predictor for feasibility of rheumatic MV repair.

\section{Discussion}

Rheumatic disease is still one of the main causes of MV dysfunction globally. RHD progresses more rapidly and it is a common phenomenon in developing countries $(1,2)$, the entire MV apparatus which is constituted of leaflets, chordae tendineae, papillary muscle and annulus can be adversely affected. Characteristic of rheumatic pathology includes (3): commissural fusion, leaflet thickening, especially at the free edges, shortening and fusion of chordae, "fish mouth" the appearance of the MV orifice. When severe pathological MV influences the hemodynamic circulation and causes patients' clinical symptom, surgical strategies are essential to treat the disease, both MV repair and MV replacement are surgical treatment for RHD patients. The outcome of MV repair for patients with RHD is outstanding and comparative with the result of repair for the degenerative MV disease (11), which is much better than rheumatic MV replacement regarding survival rate and adverse events $(4,12)$. Nevertheless, the overall rate of rheumatic MV repair is extremely low in China while the proportion of $M V$ repair in the other countries is higher $(13,14)$. Absence of indications and predictors that help surgeons select proper patients for MV repair and technique factor may be the major reasons, $M V$ repair is a promising approach for rheumatic MV disease in the proper lesions which can be reconstructed, the assessment of length and mobility and flexibility of AML is one of the crucial procedures in selecting appropriate patients. Gupta et al. (6) suggested that AML length is a predictor for MV repair in rheumatic population, their study reported that an intraoperatively measured AML length of $26 \mathrm{~mm}$ or more predicts reparability and indexed AML length which means intraoperatively measured AML length indexed to body surface area is a much stronger predictor for rheumatic MV repair, with a value of $18 \mathrm{~mm} / \mathrm{m}^{2}$ or more, repair surgery can be accomplished in all cases. In the current study, we used bending angle of AML to quantify the mobility and flexibility of AML, BT-angle in repair group was significantly larger than that of replacement group $\left(21.56^{\circ} \pm 3.84^{\circ}, 10.29^{\circ} \pm 6.02^{\circ}\right.$, respectively, $\left.\mathrm{P}<0.001\right)$, therefore the $\mathrm{BT}$-angle was tested as a predictor of reparability via observing the ROC curve (ROC area: 0.944, standard error: 0.06, 95\% CI: 0.826-1), BT-angle on TTE preoperatively of $15.5^{\circ}$ or more predicts feasibility of rheumatic MV repair with $100 \%$ sensitivity and $85.7 \%$ specificity . Commissural calcification is a very common pathological change for rheumatic MV patients, shall we directly convert rheumatic MV repair into replacement without attempt when commissural calcification is detected? The answer is we still can try to repair MV with outstanding 
result in patient with small single area (diameter $<0.8 \mathrm{~cm}$ ) of calcification at commissure and it may have no effect on reparability of rheumatic $M V$ disease ( $\beta$ : $0.08, \operatorname{Exp}(\beta)$ : 1.083, P: $0.777>0.05$ )

Wilkins scoring system (8) was established in 1988 to predict the outcome of percutaneous balloon dilatation in patient with MS. Four echocardiographic characteristics of $M V$ were scored on predilation echocardiography: leaflet mobility, leaflet thickness, subvalvular thickening and calcification. Each variable was scored 0 to 4 which the higher score represents more severe involvement, on a scale of 0 to 16 , higher score $(>11)$ was the predictor of suboptimal outcomes (15). Although Wilkins scoring system is classic and frequently used to predict the outcome of percutaneous balloon dilatation for MS, it is also an excellent assessment method of MV structure, previously there were very few studies that statistically discussed about the applicability of WS in predicting the outcome and feasibility of MV repair surgery in patients with rheumatic MS and/or MR. Our study revealed that the differences of total WS score and each component score between repair group and replacement group were not statistically significant $(\mathrm{P}>0.05)$. Through the ROC curve for total WS score (ROC area: 0.508), the WS cannot be a suitable method for pre-judging the outcome of $\mathrm{MV}$ repair in RHD patients. We consider the following probable reasons: (I) WS can assess the calcified leaflet, nonetheless, it cannot evaluate the commissural calcification and subvalvular apparatus calcification. Lesions of the commissure and subvalvular apparatus are more common pathological features in Chinese patients with rheumatic MV disease (16), WS which cannot detect the calcification of commissure and subvalvular apparatus may seriously influence the predicting accuracy if we use it to forecast the outcome of rheumatic MV repair surgery; (II) MR is considered to be associated with inadequate coaptation length which contracted AML is resulted from chronic rheumatic inflammatory, the "short AML" can lead to MV repair improbable, meanwhile, severe contracted subvalvular apparatus may diminish the feasibility of rheumatic MV repair as well. Nevertheless, the WS doesn't involve to scoring the length of AML and subvalvular apparatus. It is only applicable to isolated MS for PMBV, it is not applicable to pure MR and mixed lesions. To sum up, WS is unable to predict the feasibility of $M V$ repair in RHD patients.

$\mathrm{PMBV}$ is an effective therapeutic modality for rheumatic MS and recommended for symptomatic patients with severe MS and favorable valve morphology in the absence of atrial fibrillation (AF) (9), however, trends of overall utilization were decreasing, while cost and procedural complication rates were increasing over period of 13 years (17). How to choose procedural strategy when result of $\mathrm{PMBV}$ is uncertain? Shall we persist to conduct PMBV or alternate to rheumatic MV repair? We think the answer may be more likely to rheumatic MV repair. The reasons are: (I) unfavorable valve anatomy is the most important risk factor of long-term outcomes (18), patients who undergo PMBV must be properly selected for valve structure. WS is the most effective and extensively used evaluation system for PMBV, patients which scored $>11$ point tend to be suboptimal outcome of PMBV (8) and undergoing open valve surgery is more reasonable. However, outcomes of PMBV in Patients which scored 9-11 cannot be guaranteed (8), in-patient mortality occurred in patients in the WS gray zone (9-11) was $8.3 \%$ (19), which was significantly higher than the 30-day mortality after rheumatic MV repair that we have reported was only $1.9 \%$ (20). Valve tear and acute severe MR following PMBV are not uncommon complications for $\mathrm{PMBV}$, in-patient mortality of emergency surgery for these complications can achieve $12 \%$ (21) if we insist to carry out the intervention therapy when result of $\mathrm{PMBV}$ is uncertain, in these cases rheumatic MV repair may be the preferred surgical option. Jae-Kwan Song and his colleagues' study (22) directly compared the results of PMBV with open heart surgery and concluded that MV surgery was associated with a higher adjusted rate of long-term event-free survival than PMBV. Patients with high WS scores or AF revealed better outcomes after MV surgery. Even in the patients scored $<8$ and normal sinus rhythm which are traditionally considered to have optimal outcome for PMBV, the outcome of rheumatic MV surgery is outstanding as well as that of PMBV. Actually rheumatic $M V$ repair is a better procedural strategy than replacement in patients with feasible repair structure, outcome of $M V$ repair is widely verified better than $M V$ replacement and successful durable repairs have been performed in highvolume, expert centers (23). Severe diseased subvalvular apparatus and AF (24) can adversely affected the result of the intervention procedure, $M V$ repair is an optimal strategy for these patients even if the WS $<8$.

\section{Limitation}

The number of patients enrolled for this study was relatively small, which may adversely affect our findings for the predictive factors and success of rheumatic MV repair. 
Rheumatic MV disease is complex apparatus involvement of MV structure, study of mobility and angle of AML and commissural calcification is only one part of them, the other rheumatic MV pathological changes which may influence the outcome of rheumatic MV repair was not reflected in the study, such as subvalvular apparatus contracture, calcification, fusion, and even mitral annulus calcification and so on.

\section{Conclusions}

The bending angle of AML which was considered as quantification of mobility and flexibility of AML can be a predictor for MV repair in patient with RHD. The small single area of commissural calcification had no effect on reparability of rheumatic MV disease. WS cannot be an appropriate predictor for the outcome of rheumatic MV repair.

\section{Acknowledgements}

Funding: The study was supported by the Foundation of Beijing Anzhen Hospital, Capital Medical University (No. 2015P01) and Beijing Natural Science Foundation (No. 7174294).

\section{Footnote}

Conflicts of Interest: The authors have no conflicts of interest to declare.

Ethical Statement: This study was approved by the Ethic Committee of Beijing Anzhen Hospital of Capital Medical University (No. 2017044X).

\section{References}

1. Geldenhuys A, Koshy JJ, Human PA, et al. Rheumatic Mitral Repair Versus Replacement in a Threshold Country: The Impact of Commissural Fusion. J Heart Valve Dis 2012;21:424-32.

2. Carapetis JR. The stark reality of rheumatic heart disease. Eur Heart J 2015;36:1070-3.

3. Harb SC, Griffin BP. Mitral Valve Disease: a Comprehensive Review. Curr Cardiol Rep 2017;19:73.

4. Kim JB, Kim HJ, Moon DH, et al. Long-term outcomes after surgery for rheumatic mitral valve disease: valve repair versus mechanical valve replacement. Eur J
Cardiothorac Surg 2010;37:1039-46.

5. Waikittipong S. Mitral valve repair for rheumatic mitral regurgitation: Mid-term results. Asian Cardiovasc Thorac Ann 2015;23:658-64.

6. Gupta A, Gharde P, Kumar AS. Anterior Mitral Leaflet Length: Predictor for Mitral Valve Repair in a Rheumatic Population. Ann Thorac Surg 2010;90:1930-3.

7. Hollenberg SM. Valvular Heart Disease in Adults: Etiologies, Classification, and Diagnosis. FP Essent 2017;457:11-6.

8. Wilkins GT, Weyman AE, Abascal VM, et al. Percutaneous balloon dilatation of the mitral valve: an analysis of echocardiographic variables related to outcome and the mechanism of dilatation. Br Heart J 1988;60:299-308.

9. Nishimura RA, Otto CM, Bonow RO, et al. 2014 AHA/ ACC Guideline for the Management of Patients With Valvular Heart Disease: executive summary: a report of the American College of Cardiology/American Heart Association Task Force on Practice Guidelines. J Am Coll Cardiol 2014;63:2438-88.

10. Nishimura RA, Otto CM, Bonow RO, et al. 2017 AHA/ ACC Focused Update of the 2014AHA/ACC Guideline for the Management of Patients With Valvular Heart Disease A Report of the American College of Cardiology/ American Heart Association Task Force on Clinical Practice Guidelines. J Am Coll Cardiol 2017;70:252-89.

11. Yakub MA, Dillon J, Krishna Moorthy PS, et al. Is rheumatic aetiology a predictor of poor outcome in the current era of mitral valve repair? Contemporary longterm results of mitral valve repair in rheumatic heart disease. Eur J Cardiothorac Surg 2013;44:673-81.

12. Wang Z, Zhou C, Gu H, et al. Mitral Valve Repair versus Replacement in Patients with Rheumatic Heart Disease. J Heart Valve Dis 2013;22:333-9.

13. Kim GS, Lee CH, Kim JB, et al. Echocardiographic evaluation of mitral durability following valve repair in rheumatic mitral valve disease: Impact of Maze procedure. J Thorac Cardiovasc Surg 2014;147:247-53.

14. El Oumeiri B, Boodhwani M, Glineur D, et al. Extending the Scope of Mitral Valve Repair in Rheumatic Disease. Ann Thorac Surg 2009;87:1735-40.

15. Stehouwer N, Okello E, Gupta V, et al. Development and Validation of a Teaching Module for Echocardiographic Scoring of Rheumatic Mitral Stenosis. Glob Heart 2017. [Epub ahead of print].

16. Tiange L, Xu M. Repair Strategies Based on Pathological Characteristics of the Rheumatic Mitral Valve in Chinese 
Patients. Heart Lung Circ 2017. [Epub ahead of print].

17. Badheka AO, Shah N, Ghatak A, et al. Balloon Mitral Valvuloplasty in the United States: A 13-Year Perspective. Am J Med 2014;127;1126.e1-12.

18. Jorge E, Pan M, Baptista R, et al. Predictors of Very Late Events After Percutaneous Mitral Valvuloplasty in Patients With Mitral Stenosis. Am J Cardiol 2016;117:1978-84.

19. Paiva M, Correia AS, Lopes R, et al. Selection of patients for percutaneous balloon mitral valvotomy: Is there a definitive limit for the Wilkins score? Rev Port Cardiol 2013;32:873-8.

20. Fu JT, Popal MS, Zhang HB, et al. A meta-analysis of late outcomes of mitral valve repair in patients with rheumatic heart disease. J Thorac Dis 2017;9:4366-75.

Cite this article as: Fu JT, Popal MS, Jiao YQ, Zhang HB, Zheng S, Hu QM, Wei H, Meng X. A predictor for mitral valve repair in patient with rheumatic heart disease: the bending angle of anterior mitral leaflet. J Thorac Dis 2018;10(5):2908-2915. doi: $10.21037 /$ jtd.2018.05.26
21. Nanjappa MC, Ananthakrishna R, Hemanna Setty SK, et al. Acute severe mitral regurgitation following balloon mitral valvotomy: Echocardiographic features, operative findings, and outcome in 50 surgical cases. Catheter Cardiovasc Interv 2013;81:603-8.

22. Song JK, Kim MJ, Yun SC, et al. Long-term outcomes of percutaneous mitral balloon valvuloplasty versus open cardiac surgery. J Thorac Cardiovasc Surg 2010;139:103-10.

23. Yanagawa B, Butany J, Verma S. Update on rheumatic heart disease. Curr Opin Cardiol, 2016;31:162-8.

24. Bhalgat P, Karlekar S, Modani S, et al. Subvalvular apparatus and adverse outcome of balloon valvotomy in rheumatic mitral stenosis. Indian Heart J 2015;67:428-33. 